\title{
Is Muscle Weakness a Risk Factor of the Development of Rotator Cuff Tendinopathy - A Systematic Review
}

\section{Cheuk-Kin Kwan}

Department of Orthopaedics and Traumatology, Faculty of Medicine, The Chinese University of Hong Kong

Man-Chi Ko

Department of Orthopaedics and Traumatology, Faculty of Medicine, The Chinese University of Hong Kong

Sai-Chuen Fu

Department of Orthopaedics and Traumatology, Faculty of Medicine, The Chinese University of Hong Kong

Samuel Ka-Kin Ling

Department of Orthopaedics and Traumatology, Faculty of Medicine, The Chinese University of Hong Kong

Hio-Teng Leong

Department of Orthopaedics and Traumatology, Faculty of Medicine, The Chinese University of Hong Kong

Joo-Han Oh

Department of Orthopaedic Surgery, Seoul National University College of Medicine, Seoul National University Bundang Hospital, Seongnam

Patrick Shu-Hang Yung ( $\sim$ patrickyung@cuhk.edu.hk)

Chinese University of Hong Kong

\section{Research article}

Keywords: Tendinopathy, Rotator Cuff, Muscle Weakness, Muscle Stiffness, Risks, Systematic Review

Posted Date: November 23rd, 2020

DOI: https://doi.org/10.21203/rs.3.rs-102394/v1

License: (c) (i) This work is licensed under a Creative Commons Attribution 4.0 International License. Read Full License 


\section{Abstract}

Background

Underlying muscle weakness may explain the inconsistency in the development of rotator cuff tendinopathy in population with similar activity levels. This systematic review aims to assess existing prospective studies to evaluate whether muscle weakness is a risk factor to the development of rotator cuff tendinopathy.

Methods

A systematic search was performed using the PRISMA guidelines. Prospective studies measuring muscle strength or stiffness and the incidence of rotator cuff tendinopathy will be included. Quality assessment was performed with the Newcastle-Ottawa Quality Assessment Scale.

Results

The search yielded 6 studies, with a total of 523 trained overhead athletes followed up for 1 season. External and internal rotation strength was described as protective factors for the development of rotator cuff tendinopathy, with an odds ratio of $0.940(p<0.05)$ and $0.946(p<0.01)$ respectively for each N/m increased in force generated. Limited range of motion of $<106^{\circ}$ for shoulder external rotation was also described as a risk factor with an odds ratio of 1.12 ( $p<$ 0.001).

Imbalance between external and internal rotation strength was reported as a risk factor for shoulder injuries in 2 studies, with a relative risk of 2.57 ( $p<0.05$ ) reported in 1 of them. Supraspinatus weakness was also reported as a risk factor for shoulder injuries in 1 study, with no odds ratio or relative risk provided.

Due to insufficient data, combination into a meta-analysis was not possible.

\section{Conclusions}

Limited evidence support that weakness of the external rotators, weakness in the internal rotators, and limited range of motion in external rotation are risk factors to the development of rotator cuff tendinopathy. Very limited evidence support that imbalance in external rotator and internal rotator strength, and supraspinatus weakness are risk factors for rotator cuff tendinopathies. Future cohort studies may improve on existing evidence with investigations on more muscle groups, a longer follow-up time, clearly documented injury history, and a stringent diagnosis to rotator cuff tendinopathy.

\section{Background}

Rotator cuff tendinopathy is an umbrella term describing pain and loss of function in the rotator cuff tendon. Entities including tendinosis, tendinitis, subacromial

impingement, subacromial bursitis are often regarded under the term rotator cuff tendinopathy.(1) As reported in previous studies, rotator cuff tendinopathy affects more than $50 \%$ of the population over the age of 60 , contributing to the loss of function and poor quality of life.(2) As a consequence, rotator cuff tendinopathy also leads to large socioeconomical impacts.(3)

The pathogenesis of tendinopathy is believed to involve a failed healing process, of which etiology is multifactorial(4). Overload or overuse of the rotator cuff tendons was suggested to be one of the factors leading to the development of rotator cuff tendinopathy. To explain the association of tendinopathy with overuse, previous studies have demonstrated that tendon loading, in terms of compression or tensile loads, would induce apoptosis and inflammation to tendon cells.(5,6) An increased activity level would increase tendon loading. However, the fact that athletes with similar training load present inconsistently with tendinopathy suggest that some factors may play a role in the modulation of tendon loading under given training load.

Muscle weakness may be a factor modulating tendon loading. It is a relative term referring to the failure to generate a desired or expected force.(7) The mechanism of how weakness in rotator cuff muscles may lead to rotator cuff tendinopathies has been hypothesized by multiple groups. As suggested by previous studies, rotator cuff muscles prevent humeral head elevation, which will decrease the size of subacromial space and increase the risk of supraspinatus impingement.(8),(9) The reduction of subacromial space in patients with rotator cuff tendinopathy was also demonstrated by a previous retrospective study.(10) Another study suggested that external rotation strength is crucial for the deceleration of arm swinging movements.(11) Its weakness in athletes with repetitive forceful arm swings is hypothesized lead to strains and tears to the tendon.(12)

Muscle stiffness was also suggested to lead to weaken muscles via reciprocal inhibition from a tight antagonist(8). The stiffness of upper trapezius muscles was demonstrated in patients with rotator cuff tendinopathy in a previous retrospective study(13). There is a possibility that muscle stiffness of the shoulder may be one of the causes leading to muscle weakness and the development of rotator cuff tendinopathies.

In summary, expert opinions have suggested that muscle weakness and stiffness may lead to an increased risk of the development of tendinopathies.(14,15) This suggestion has been supported by several authors reviewing retrospective and cadaveric studies.(10,16-19) However, as these parameters are readily influenced by the presence of pain, the association between muscle imbalance and the development of tendinopathy is yet to be established.

\section{Purpose of this review}

This review aims to examine existing prospective studies on identifying the association between muscle weakness and rotator cuff tendinopathy. Muscle weakness would be identified by direct measurements of muscle strength, while associated factors like muscle stiffness may suggest a cause to the 
underlying muscle weakness.

\section{Methods}

Systemic search was carried out in PubMed, CINAHL, and Scopus from inception to May $13^{\text {th }}, 2020$. The Preferred Reporting Items for Systematic Reviews and Meta-Analyses (PRISMA) statements was used as guidelines in the performance of the systematic review.(20) Our study have been registered to the PROSPERO database, under the registration number CRD42020197927. The searched keywords are shown in Table I. Supplementary searches were carried out by hand searching the reference lists of the included studies.

\section{Table I. Search terms used}

\begin{tabular}{|ll|}
\hline $\mathbf{1}$ & Cohort \\
\hline $\mathbf{2}$ & Prospective \\
\hline $\mathbf{3}$ & 1 OR 2 \\
\hline $\mathbf{5}$ & Tendinopathy \\
\hline $\mathbf{6}$ & Tendon \\
\hline $\mathbf{7}$ & Tear \\
\hline $\mathbf{8}$ & Injury \\
\hline $\mathbf{9}$ & Pathology \\
\hline 10 & 6 OR 7 OR 8 OR 9 \\
\hline 11 & 5 AND 10 \\
\hline $\mathbf{1 2}$ & Subacromial \\
\hline $\mathbf{1 3}$ & Impingement \\
\hline $\mathbf{1 4}$ & Bursitis \\
\hline $\mathbf{1 5}$ & 13 OR 14 \\
\hline $\mathbf{1 6}$ & 12 AND 15 \\
\hline $\mathbf{1 7}$ & $\mathbf{4}$ OR 11 OR 16 \\
\hline $\mathbf{1 8}$ & Weakness \\
\hline $\mathbf{1 9}$ & Muscle weak* \\
\hline $\mathbf{2 0}$ & Deficit \\
\hline $\mathbf{2 1}$ & Strength \\
\hline $\mathbf{2 2}$ & Stiffness \\
\hline $\mathbf{2 3}$ & Flexibility \\
\hline
\end{tabular}

\section{Eligibility criteria}

Clinical studies were included if ALL the followings are met (i) prospective data containing the measurement of muscle strength or muscle stiffness were documented. (ii) Incidence of tendinopathy was documented. (iii) Patients were described to have rotator cuff tendinopathy / tendinosis / tendinitis / tear, or subacromial impingement / bursitis. A study was excluded if (i) patients were treated with any interventions, and (ii) the study was an animal study.

\section{Study selection and data collection}

Studies from the systematic search were merged in EndNote X9 (Thomson, New York), with duplicates removed. Application of exclusion and inclusion criteria was performed by screening the titles and abstracts, followed by retrieval of full texts of included studies. Two reviewers (CKK and MCK) independently screened all the titles and abstracts, and reviewed the identified studies for inclusion. Disagreements were resolved by consensus between 2 reviewers. A third reviewer (FSC) was available to resolve further disagreements. A PRISMA-flowchart of the selection process is shown in Fig. 1. 
Newcastle-Ottawa Quality Assessment Scale was used to assess the quality of included studies. Out of a full score of 9 , studies scoring $\geq 7$ indicates high methodological quality, 6 indicates intermediate quality, and $\leq 5$ indicates low quality. The assessment of study quality was performed in a standardized manner independently by two reviewers (CKK, MCK). Disagreements were resolved by consensus between the 2 reviewers. A third reviewer (FSC) was available to resolve further disagreements. Intra-class correlation coefficient (ICC) 2-way mixed-effects analysis was calculated using SPSS Version 20 for Windows (SPSS Inc., Chicago, IL) to measure the inter-rater agreement between 2 reviewers for the quality assessment.

Results

\begin{tabular}{|c|c|c|c|c|c|c|c|c|c|c|c|c|}
\hline \multirow[t]{2}{*}{ Study } & \multirow[t]{2}{*}{ Quality } & \multirow{2}{*}{$\begin{array}{l}\text { Sample } \\
\text { size }\end{array}$} & \multirow{2}{*}{$\begin{array}{l}\text { Female } \\
\text { ratio } \\
(\%)\end{array}$} & \multirow[t]{2}{*}{ Sport* } & \multirow[t]{2}{*}{$\mathrm{Age}^{\star \star}$} & \multirow{2}{*}{$\begin{array}{l}\text { Diagnostic } \\
\text { method }\end{array}$} & \multirow{2}{*}{$\begin{array}{l}\text { Total } \\
\text { cases of } \\
\text { shoulder } \\
\text { injury } \\
\text { (Cases } \\
\text { with a } \\
\text { hx of } \\
\text { previous } \\
\text { injury) }\end{array}$} & \multirow{2}{*}{$\begin{array}{l}\text { Cases of } \\
\text { tendinopathy*** }\end{array}$} & \multicolumn{4}{|c|}{ Risk factor associated with shoulder injı } \\
\hline & & & & & & & & & $\begin{array}{l}\text { Low IR } \\
\text { strength }\end{array}$ & $\begin{array}{l}\text { Low ER } \\
\text { strength }\end{array}$ & $\begin{array}{l}\text { Low } \\
\text { ER/IR } \\
\text { strength } \\
\text { ratio }\end{array}$ & Othe \\
\hline $\begin{array}{l}\text { Forthomme } \\
\text { 2018(21) }\end{array}$ & $7 / 9$ & 106 & 0 & Handball & $\begin{array}{l}\text { Young } \\
\text { adults }\end{array}$ & $\begin{array}{l}\text { Not } \\
\text { specified }\end{array}$ & $23(n / a)$ & 15 & - & - & - & \\
\hline $\begin{array}{l}\text { Edouard } \\
\text { 2013(22) }\end{array}$ & $7 / 9$ & 16 & 100 & Handball & $\begin{array}{l}\text { Young } \\
\text { adults }\end{array}$ & $\begin{array}{l}\text { Not } \\
\text { specified }\end{array}$ & $9(6)$ & 6 & - & - & + & \\
\hline $\begin{array}{l}\text { Forthomme } \\
2013(23)\end{array}$ & $8 / 9$ & 66 & 48 & Volleyball & $\begin{array}{l}\text { Young } \\
\text { adults }\end{array}$ & $\begin{array}{l}\text { Clinical } \\
\text { with } \\
\text { radiology }\end{array}$ & $15(13)$ & 15 & + & + & - & \\
\hline $\begin{array}{l}\text { wang } \\
2001(24)\end{array}$ & $5 / 9$ & 16 & 0 & Volleyball & $\mathrm{n} / \mathrm{a}$ & $\begin{array}{l}\text { Not } \\
\text { specified }\end{array}$ & $7(n / a)$ & 3 & $\mathrm{n} / \mathrm{a}$ & - & + & \\
\hline $\begin{array}{l}\text { Byram } \\
2010(25)\end{array}$ & $5 / 9$ & 207 & 0 & Baseball & $\mathrm{n} / \mathrm{a}$ & $\begin{array}{l}\text { Not } \\
\text { specified }\end{array}$ & $41(n / a)$ & 21 & - & + & - & $\begin{array}{l}\text { Supri } \\
\text { weak }\end{array}$ \\
\hline $\begin{array}{l}\text { Polster } \\
\text { 2016(26) }\end{array}$ & $8 / 9$ & 112 & 0 & Baseball & Adults & $\begin{array}{l}\text { Clinical } \\
\text { with } \\
\text { radiology }\end{array}$ & $10(n / a)$ & 10 & $\mathrm{n} / \mathrm{a}$ & $\mathrm{n} / \mathrm{a}$ & $\mathrm{n} / \mathrm{a}$ & $\begin{array}{l}\text { ER ra } \\
\text { motic }\end{array}$ \\
\hline
\end{tabular}

\section{Table II. Summary of extracted data}

*All studies included athletes with a training load of $>7$ hours/ week

**Age: 18-30 young adult, 30-40 adult, 40-60 middle age, $>60$ elderly

*** Cases of tendinopathy are included in the "total shoulder injuries" column

**** IR: internal rotation, ER, external rotation, + : factor is reported to be a significant risk factor, -: factor is reported to be an insignificant factor, $\mathrm{n} / \mathrm{a}$ : factor not assessed in the study

\section{Quality Assessment}

\begin{tabular}{|c|c|c|c|c|c|c|c|c|c|c|}
\hline Study & Total Score & 1 & 2 & 3 & 4 & 5 & 6 & 7 & 8 & 9 \\
\hline Forthomme 2018(21) & 7 & + & + & + & - & + & + & - & + & + \\
\hline Edouard 2013(22) & 7 & - & + & + & - & + & + & + & + & + \\
\hline Forthomme 2013(23) & 8 & + & + & + & - & + & + & + & + & + \\
\hline Wang 2001(24) & 5 & - & + & + & - & + & - & - & + & + \\
\hline Byram 2010(25) & 5 & + & + & + & - & + & - & - & + & - \\
\hline Polster 2016(26)* & 8 & + & + & + & + & + & - & + & + & + \\
\hline
\end{tabular}

Table III. Methodological quality assessment using the Newcastle-Ottawa Scale for Cohort Studies

+: point given for this category $\quad$-: point not given for this category

The Newcastle-Ottawa Scale for Cohort Studies 


\begin{tabular}{|c|c|c|c|c|c|}
\hline 1 & $\begin{array}{l}\text { Representativeness of the } \\
\text { exposed cohort }\end{array}$ & 4 & $\begin{array}{l}\text { Demonstration that outcome of interest was not present at } \\
\text { start of study }\end{array}$ & 7 & Assessment of outcome \\
\hline 2 & $\begin{array}{l}\text { Selection of the non exposed } \\
\text { cohort }\end{array}$ & 5 & Study controls for the most important factor & 8 & $\begin{array}{l}\text { Was follow-up long enough for } \\
\text { outcomes to occur }\end{array}$ \\
\hline 3 & Ascertainment of exposure & 6 & Study controls for additional factors & 9 & Adequacy of follow up of cohorts \\
\hline
\end{tabular}

* The Newcastle-Ottawa Scale for Case Control Studies

\begin{tabular}{|llllll|}
\hline 1 & Is the case definition adequate? & 4 & Definition of Controls & 7 & Ascertainment of exposure \\
\hline 2 & Representativeness of the cases & 5 & Study controls for the most important factor & 8 & Same method of ascertainment for cases and controls \\
\hline 3 & Selection of Controls & 6 & Study controls for additional factors & 9 & Non-Response rate \\
\hline
\end{tabular}

\section{Results}

\section{Studies included}

The systematic search resulted in 13,789 studies, which 663 were suitable for abstract review. Results were further screened down to 12 studies for full text review. Six studies were included while the remainder were excluded as the entity of investigation was not rotator cuff tendinopathy, or prospective measurements of muscle weakness was not documented.

Six included studies prospectively documented muscle weakness or stiffness, and the incidence of rotator cuff tendinopathy in a total of 523 patients. 105 patients developed shoulder injuries during the follow-up period, of which 70 were diagnosed as rotator cuff tendinopathy. However, statistical association between muscle weakness or stiffness and the incidence of tendinopathy was only calculated in two out of the six studies(23,26). The other four demonstrated the association between muscle weakness or stiffness against shoulder injuries in general $(21,22,24,25)$. The documentation of previous injuries were also inconsistent. Of the two studies which documented for previous injuries, 19 out of 24 cases of shoulder injuries had an injury history. All included studies had a follow-up period of 1 season, approximately 6 months depending on the schedule of specific leagues. The studied population of all 6 studies were trained overhead athletes participating in either volleyball $(23,24)$, baseball $(25,26)$, or handball $(21,22)$. Exact training load, in terms of hours per week, was not comparable due to inconsistent documentation among studies. Participants were free of shoulder pain at the beginning of studies, but other aspects of the past medical history were not documented. The diagnosis of tendinopathy also varied among the studies. Four studies did not provide the diagnostic criteria of rotator cuff tendinopathy $(21,22,24,25)$, while 2 studies described the diagnosis to be made clinically and confirmed with radiological evidence. $(23,26)$

\section{Methodological quality assessment}

The results of quality assessment with the Newcastle-Ottawa Scale for Cohort Studies are included in Table II. Among 6 included studies, there were 4 of high methodological quality, and 2 with low quality. The criteria regarding the demonstration of an absence of the outcome of interest at the start of the study was not achieved in all included studies, as the absence of rotator cuff tendinopathy was only recorded from self-reporting questionnaires, without the support of clinical or radiological testing. The intra-class correlation coefficient for the inter-rater agreement between 2 reviewers was 0.960 ( $95 \% \mathrm{Cl} 0.733-0.994$ ).

\section{Studies reporting rotator cuff strength and stiffness as risk factors to the development of rotator cuff tendinopathy}

One included study discussed isometric internal and external rotation strength (IR \& ER), with an isokinetic dynamometer, as a possible risk factor to the development of rotator cuff tendinopathy.

ER strength was described as a protective factor. An odds ratio of $0.940(p<0.05)$ for each $1 \mathrm{~N} / \mathrm{m}$ increased in maximal force generated eccentrically at $60^{\circ}$ of shoulder abduction(23).

IR strength was also described as a protective factor. An odds ratio of $0.946(p<0.01)$ was reported for each $\mathrm{N} / \mathrm{m}$ increased in maximal force generated eccentrically at $60^{\circ}$ of shoulder abduction (23)

The significance of ER range of motion, hand measured with a goniometer, was reported in one study(26). An odds ratio of 1.12 ( $p<0.001)$ was reported for a dominant arm ER range of $<106^{\circ}$.

Due to insufficient number of studies, combination into a meta-analysis was not possible. The authors were contacted in request for necessary raw data for calculation, but no additional data were provided.

\section{Studies reporting rotator cuff strength and stiffness as risk factors to the development of shoulder injuries}

Five studies discussed isometric rotator cuff strength, with an isokinetic dynamometer, as a possible risk factor to the development of shoulder injuries, of which 70 out of 105 cases were diagnosed as rotator cuff tendinopathies.

On top of the studies mentioned in the previous paragraph, Imbalance between ER and IR strength was mentioned in two studies. However, the definition for muscle imbalance varied. In one study, imbalance was defined as a significant strength difference between IR and ER strength, with no relative risk or odds 
ratio provided.(24) In the other study, a cut off of $<69 \%$ of IR/ER ratio was adapted, and a relative risk of 2.57 ( $p<0.05$ ) was reported for athletes with muscle imbalance.(22)

ER and supraspinatus weakness was reported in one study with no odds ratio or relative risk provided.(25)

Due to insufficient number of studies, combination into a meta-analysis was not possible. The authors were contacted in request for necessary raw data for calculation, but no additional data were provided.

\section{Discussion}

According to our systematic search, IR weakness, ER weakness, and limited range of ER at the glenohumeral joint have been reported to be associated with the development of rotator cuff tendinopathy. Supraspinatus weakness and imbalance in IR/ER strength have also been reported as a risk factor in the development of shoulder injuries.

\section{Limited evidence support that weakness and stiffness of the rotator cuff muscles are risk factors to the development of rotator cuff tendinopathy}

Our findings showed the significance of pre-injury muscle strength and stiffness on the incidence of tendinopathy. The reported risk factors include weakness of the shoulder external rotators, weakness of the internal rotators, and a small range of motion for external rotation at the glenohumeral joint. It was demonstrated in previous studies that these muscle changes, in the absence of pain, may increase the risk of newly developing rotator cuff tendinopathies in the upcoming season.

Other risk factors such as a low IR/ER ratio and supraspinatus weakness were also mentioned in included studies. Although the significance of these factors were associated with shoulder injuries, rotator cuff tendinopathies make up 70 out of 105 total cases of shoulder injuries included. There is a possibility that the risk factors may also be valid for the development of rotator cuff tendinopathy, but the calculations were unfortunately not commenced in our included studies(22,24,25).

Risk factors were classified into five evidence levels in a previous systematic review on rotator cuff tendinopathy. $(27,28)$ Using the same method, evidence supporting that weakness and stiffness of rotator cuff muscles are risk factors to the development of rotator cuff tendinopathy could be classified as limited evidence. Very limited evidence support that a low IR/ER ratio and supraspinatus weakness are risk factors to the development of rotator cuff tendinopathy.

\section{Hypothesis on how muscle weakness may lead to rotator cuff tendinopathy}

Tendon loading, in terms of tension or compression to the tendon, is one of the risk factors leading the development to tendinopathy. this suggestion is supported by previous in vitro studies, demonstrating that tension or compression of tendon cells will lead to apoptosis and increased expression of inflammatory markers. $(5,6,29)$ As mentioned in the introduction, several mechanisms have been proposed by various groups to explain how muscle weakness may lead to increased loading. Strength in the rotator cuff muscles are crucial in counteracting the deltoid muscle, keeping the humeral head adhered to the glenoid.(8) with weakened or fatigued rotator cuff muscles, humeral head elevation may occur, decreasing the size of sub-acromial space, leading to compression of the supraspinatus tendon. $(9,10)$ Another possible mechanism was described in a previous study suggesting that IR and ER strength is crucial for the deceleration of arm swinging movements.(11) It was illustrated that the requirement for ER strength is increased to effectively decelerate an arm from forceful IR. Imbalance in athletes with repetitive arm swings is hypothesized lead to strains and tears to the tendon.(12)

\section{Muscle weakness may not be an isolated problem}

Associated factors such as muscle stiffness posture, and movement patterns may provide more insights on the causes of muscle weakness, as well as a direction on how to effectively correct it. Unfortunately, it was demonstrated in our systematic search that the association of these factors to the development of rotator cuff tendinopathy is under-investigated, with only one existing study discussing the significance of shoulder range of motion to the development of rotator cuff tendinopathy.(26) This study demonstrated that limited ER range of the shoulder is associated with the development of rotator cuff tendinopathy. Unfortunately, whether range of motion was active or passively measured was not described. This piece of information suggests a cause of the underlying muscle weakness. Limited range in ER may imply stiffness of internal rotators such as pectoralis major, teres major, and subscapularis, latissimus dorsi, and deltoid muscles. Considering the concept of reciprocal inhibition, tightness in this muscle group would lead to weakness in ER muscles, which is also consistent with our result demonstrating that a weakened ER compared with IR strength is associated with the development of rotator cuff tendinopathy.(8) This finding provide evidence that muscle weakness is not the only muscle change which may lead to the development of rotator cuff tendinopathy.

\section{Could the history of a previous injury be a confounding factor}

As shown in Table 1, the history of previous injuries were inconsistently recorded in the included studies. Of the two studies which documented for injury history, 19 out of 24 cases of shoulder injury also had a history of previous shoulder injury. However, current evidence is inconclusive whether the history of a previous shoulder injury may act as a confounding factor between rotator cuff weakness and the development of rotator cuff tendinopathies.

Rotator cuff tendinopathies are described to be persistent and recurring.(30) However, A previous systematic review on the risk factors on the development of rotator cuff tendinopathy have not been able to identify existing studies on whether a previous injury history is a risk factor to the development of future rotator cuff tendinopathies.(27) Also, rotator cuff tendinopathies are well known to present as shoulder weakness(31), but little is known on whether a previous injury would lead to residual weaknesses. 
One of our included studies have attempted to address this issue.(21) According to this study, $47 \%$ of 108 athletes were documented for previous shoulder injury. However, it was shown in the study that previous injury was not a significant risk factor to development of new injury ( $p=0.28$ ). There were also no significant difference in isokinetic results between players with and without previous injuries $(p>0.05)(21)$. The significance of previous injuries were not assessed in the other included studies.

\section{Clinical relevance}

Rotator cuff tendinopathy affects a huge population, leading to disability and heavy socioeconomical impacts.(3) The results from this study provided an insight on how we may attempt to decrease such burden via primary prevention. It was demonstrated that not only in absolute strength, but imbalance in external and IR strength of the shoulder may contribute to the development of rotator cuff tendinopathy. Therefore, screening programs can be conducted in high risk groups, such as athletes competing in overhead sports, to identify individuals who are at a further high risk of the development of rotator cuff tendinopathy, and indicated for correction of muscle imbalance. There is a possibility that correction of muscle imbalance in existing cases of tendinopathy may also improve symptom control by modifying mechanical load to the tendon. However, its effectiveness on symptom control is yet to be investigated.

Regarding the method to correct muscle imbalance, it is to be acknowledged that a successful protocol would include, but is not limited to training of the weakened muscle. As mentioned in previous sections, muscle tightness would lead to reciprocal inhibition of the antagonist muscle. Attempts to relief muscle stiffness may augment the effectiveness of training to restore muscle imbalances. This provides a rationale to some existing treatments to tendinopathy, including eccentric exercises to train weak muscles, soft tissue release to relax tightened muscles. Systematic reviews on the effectiveness of these treatments alone have shown promising but inconclusive results on symptom relief of tendinopathy. $(14,32,33)$ However, the effectiveness of combined treatment of muscle relaxation followed by training have not been investigated in rotator cuff tendinopathies, which opens a direction of future clinical trials.

\section{Limitations}

All included cohort studies recruited patients in the athletic population. Therefore, the significance of muscle imbalance on the development of rotator cuff tendinopathy in the general public is currently unclear.

Rotator cuff tendinopathy observed in the included studies also presented in a rather sub-acute manner, developing in one season time. In comparison, tendinopathy in the general public is more known as a chronic degenerative disease with failed healing.(4) One explanation can be that weakness in the rotator cuff leads to an unfavorable mechanical environment, causing subclinical microtraumas chronically. Tendinitis would then develop as an acute on chronic condition from triggers such as an intense competitive season.

This hypothesis is particularly relevant as participants in the included cohort studies were painless at the beginning of follow-up, but no confirmatory imaging tests were done to confirm the absence of tendinopathy. Muscle weakness or stiffness could therefore be a result of adaptation to painless cases of tendinopathy, which its existence was previously described in an MRI study.(34) The diagnostic criteria of rotator cuff tendinopathy was also not clearly documented in several included studies $(21,22,24,25)$, providing a potential source of inconsistency.

Athletes in the included studies also varied in age, gender, and sports participated. These factors may explain the inconsistencies between the results of our included studies.

\section{Future study}

With a goal to develop screening programs and lower injury risk, future studies could be directed in three steps. To further investigate and provide more details on the profile of muscle weaknesses that may lead to rotator cuff tendinopathy, to investigate on the most effective method to correct muscle weakness, and at last to investigate whether correction of muscle weakness would lead to a decrease in injury risk.

Unfortunately, existing evidence is still far from achieving step one. The significance of muscle weakness or stiffness have only been investigated on rotator cuff muscles, while there have been hypothesis that muscles of the spine, chest, and back may also have an impact on shoulder injuries.(35) Results from this review have also pointed out several limitations on the existing cohort studies, and that a future cohort could further improve on the existing evidence with a longer follow-up time, clearly documented injury history, and a stringent diagnosis to rotator cuff tendinopathy.

Prospective investigations on muscle stiffness, posture, and movement patterns may also be fruitful, as it may provide a cause to the existing muscle imbalance, as well as provide an insight on how this problem could be more effectively corrected.

\section{Conclusions}

Limited evidence support that weakness of the external rotators, weakness in the internal rotators, and limited range of motion in external rotation are risk factors to the development of rotator cuff tendinopathy. Very limited evidence support that imbalance in external rotator and internal rotator strength, and supraspinatus weakness are risk factors for rotator cuff tendinopathies. Existing evidence on whether muscle weakness is a risk factor to the development of rotator cuff tendinopathy is limited, despite having great potentials in providing insights on the development of screening programs to lower the risk of this disease. Future cohort studies may improve on existing evidence with investigations on more muscle groups, a longer follow-up time, clearly documented injury history, and a stringent diagnosis to rotator cuff tendinopathy.

\section{Abbreviations}

IR: Internal rotation; ER: External rotation; ICC: Intra-class correlation coefficient; PRISMA: Preferred Reporting Items for Systematic Reviews and Meta-Analyses 


\section{Declarations}

\section{Acknowledgements}

Not applicable

\section{Authors' contributions}

CKK designed the study with supervision from SCF. CKK and MCK performed data collection and analysis with supervision from SCF. CKK drafted the text with supervision from SCF. SKKL, HTL, JHO, and PSHY read, and approved the various drafts up to the final manuscript

\section{Funding}

The research did not receive any specific grant from funding agencies in the public, commercial, or not-for-profit sectors, and no material support of any kind was received

\section{Availability of data and materials}

The datasets used and/or analyzed during the current study are available from the corresponding author on reasonable request.

\section{Ethics approval and consent to participate}

Not applicable

\section{Consent for publication}

Not applicable

\section{Competing interests}

The authors declare that they have no competing interests

\section{References}

1. de Oliveira FCL, Bouyer LJ, Ager AL, Roy J-S. Electromyographic analysis of rotator cuff muscles in patients with rotator cuff tendinopathy: A systematic review. J Electromyogr Kinesiol. 2017 Aug;35:100-14.

2. Seitz AL, McClure PW, Finucane S, Boardman ND, Michener LA. Mechanisms of rotator cuff tendinopathy: intrinsic, extrinsic, or both? Clin Biomech (Bristol, Avon). 2011 Jan;26(1):1-12.

3. Hopkins C, Fu S-C, Chua E, Hu X, Rolf C, Mattila VM, et al. Critical review on the socio-economic impact of tendinopathy. Asia Pac J Sports Med Arthrosc Rehabil Technol. 2016 Apr 22;4:9-20.

4. Fu S-C, Rolf C, Cheuk Y-C, Lui PP, Chan K-M. Deciphering the pathogenesis of tendinopathy: a three-stages process. Sports Med Arthrosc Rehabil Ther Technol. 2010 Dec 13;2:30.

5. Chen W, Deng Y, Zhang J, Tang K. Uniaxial repetitive mechanical overloading induces influx of extracellular calcium and cytoskeleton disruption in human tenocytes. Cell Tissue Res. 2015 Feb;359(2):577-87.

6. Chen H, Chen L, Cheng B, Jiang C. Cyclic mechanical stretching induces autophagic cell death in tenofibroblasts through activation of prostaglandin E2 production. Cell Physiol Biochem. 2015;36(1):24-33.

7. Milner-Brown HS, Miller RG. Increased Muscular Fatigue in Patients With Neurogenic Muscle Weakness: Quantification and Pathophysiology. :6.

8. Page P, Frank C, Lardner R. Assessment and Treatment of Muscle Imbalance: The Janda Approach. Human Kinetics $10 \%$; 312 p.

9. Chen SK, Simonian PT, Wickiewicz TL, Otis JC, Warren RF. Radiographic evaluation of glenohumeral kinematics: a muscle fatigue model. J Shoulder Elbow Surg. 1999 Feb;8(1):49-52.

10. Leong HT, Tsui SSM, Ng GY-F, Fu SN. Reduction of the subacromial space in athletes with and without rotator cuff tendinopathy and its association with the strength of scapular muscles. J Sci Med Sport. 2016 Dec;19(12):970-4.

11. Hess SA, Richardson C, Darnell R, Friis P, Lisle D, Myers P. Timing of rotator cuff activation during shoulder external rotation in throwers with and without symptoms of pain. J Orthop Sports Phys Ther. 2005 Dec;35(12):812-20.

12. Meister K. Injuries to the shoulder in the throwing athlete. Part one: Biomechanics/pathophysiology/classification of injury. Am J Sports Med. 2000 Apr;28(2):265-75

13. Leong HT, Hug F, Fu SN. Increased Upper Trapezius Muscle Stiffness in Overhead Athletes with Rotator Cuff Tendinopathy. PLoS ONE. 2016;11(5):e0155187.

14. Stanish WD, Curwin S, Mandell S. Tendinitis: Its Etiology and Treatment. OUP Oxford; 2000. 158 p.

15. Khan K, Brukner P. Brukner \& Khan's Clinical Sports Medicine. McGraw-Hill Education; 2011. 1296 p.

16. van der Worp H, van Ark M, Roerink S, Pepping G-J, van den Akker-Scheek I, Zwerver J. Risk factors for patellar tendinopathy: a systematic review of the literature. Br J Sports Med. 2011 Apr;45(5):446-52. 
17. Mucha MD, Caldwell W, Schlueter EL, Walters C, Hassen A. Hip abductor strength and lower extremity running related injury in distance runners: A systematic review. J Sci Med Sport. 2017 Apr;20(4):349-55.

18. Steinberg N, Dar G, Dunlop M, Gaida JE. The relationship of hip muscle performance to leg, ankle and foot injuries: a systematic review. Phys Sportsmed. 2017;45(1):49-63.

19. Lersch C, Grötsch A, Segesser B, Koebke J, Brüggemann G-P, Potthast W. Influence of calcaneus angle and muscle forces on strain distribution in the human Achilles tendon. Clinical Biomechanics. 2012 Nov 1;27(9):955-61.

20. Liberati A, Altman DG, Tetzlaff J, Mulrow C, Gøtzsche PC, loannidis JPA, et al. The PRISMA Statement for Reporting Systematic Reviews and MetaAnalyses of Studies That Evaluate Health Care Interventions: Explanation and Elaboration. PLOS Medicine. 2009 Jul 21;6(7):e1000100.

21. Forthomme B, Croisier J-L, Delvaux F, Kaux J-F, Crielaard J-M, Gleizes-Cervera S. Preseason Strength Assessment of the Rotator Muscles and Shoulder Injury in Handball Players. J Athl Train. 2018 Feb;53(2):174-80.

22. Edouard P, Degache F, Oullion R, Plessis J-Y, Gleizes-Cervera S, Calmels P. Shoulder Strength Imbalances as Injury Risk in Handball. International Journal of Sports Medicine. 2013 Feb 26;34(07):654-60.

23. Forthomme B, Wieczorek V, Frisch A, Crielaard J-M, Croisier J-L. Shoulder Pain among High-Level Volleyball Players and Preseason Features. Medicine \& Science in Sports \& Exercise. 2013 Oct; $45(10): 1852-1860$.

24. Wang H-K, Cochrane T. Mobility impairment, muscle imbalance, muscle weakness, scapular asymmetry and shoulder injury in elite volleyball athletes. The Journal of sports medicine and physical fitness. 2001 Sep 1;41:403-10.

25. Byram IR, Bushnell BD, Dugger K, Charron K, Harrell FE, Noonan TJ. Preseason Shoulder Strength Measurements in Professional Baseball Pitchers: Identifying Players at Risk for Injury. The American Journal of Sports Medicine. 2010 Jul;38(7):1375-82.

26. Polster JM, Bullen J, Obuchowski NA, Bryan JA, Soloff L, Schickendantz MS. Relationship Between Humeral Torsion and Injury in Professional Baseball Pitchers. American Journal of Sports Medicine. 2013 Sep;41(9):2015-21.

27. Leong HT, Fu SC, He X, Oh JH, Yamamoto N, Hang S. Risk factors for rotator cuff tendinopathy: A systematic review and meta-analysis. J Rehabil Med. 2019 Oct 4;51(9):627-37.

28. van Tulder M, Furlan A, Bombardier C, Bouter L, Editorial Board of the Cochrane Collaboration Back Review Group. Updated method guidelines for systematic reviews in the cochrane collaboration back review group. Spine. 2003 Jun 15;28(12):1290-9.

29. Wang JH-C, Jia F, Yang G, Yang S, Campbell BH, Stone D, et al. Cyclic mechanical stretching of human tendon fibroblasts increases the production of prostaglandin E2 and levels of cyclooxygenase expression: a novel in vitro model study. Connect Tissue Res. 2003;44(3-4):128-33.

30. Lewis JS. Rotator cuff tendinopathy/subacromial impingement syndrome: is it time for a new method of assessment? British Journal of Sports Medicine. 2009 Apr 1;43(4):259-64.

31. Neer CS. Impingement Lesions. Clinical Orthopaedics and Related Research®. 1983 Mar;173:70-77.

32. Joseph MF, Taft K, Moskwa M, Denegar CR. Deep friction massage to treat tendinopathy: a systematic review of a classic treatment in the face of a new paradigm of understanding. J Sport Rehabil. 2012 Nov;21(4):343-53.

33. Stefansson SH, Brandsson S, Langberg H, Arnason A. Using Pressure Massage for Achilles Tendinopathy: A Single-Blind, Randomized Controlled Trial Comparing a Novel Treatment Versus an Eccentric Exercise Protocol: Orthopaedic Journal of Sports Medicine [Internet]. 2019 Mar 21 [cited 2020 Feb 18]; Available from: https://journals.sagepub.com/doi/10.1177/2325967119834284

34. Connor PM, Banks DM, Tyson AB, Coumas JS, D'Alessandro DF. Magnetic resonance imaging of the asymptomatic shoulder of overhead athletes: a 5year follow-up study. Am J Sports Med. 2003 Oct;31(5):724-7.

35. Theisen C, van Wagensveld A, Timmesfeld N, Efe T, Heyse TJ, Fuchs-Winkelmann S, et al. Co-occurrence of outlet impingement syndrome of the shoulder and restricted range of motion in the thoracic spine-a prospective study with ultrasound-based motion analysis. BMC Musculoskelet Disord. 2010 Jun 29;11:135.

\section{Figures}




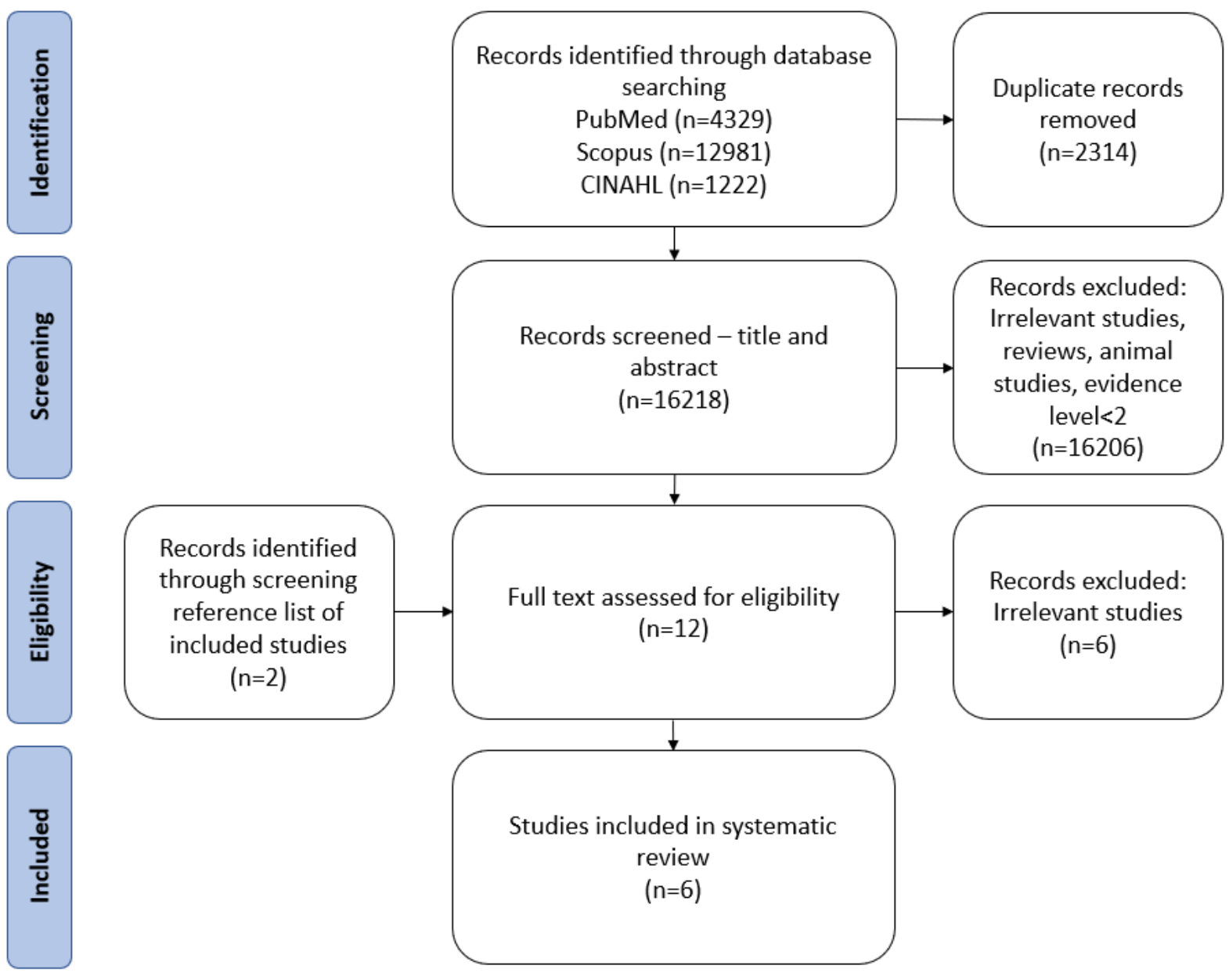

Figure 1

Preferred Reporting Items for Systematic Reviews and Meta-analyses (PRISMA) flow diagram of the search strategy is shown.

\section{Supplementary Files}

This is a list of supplementary files associated with this preprint. Click to download.

- PRISMA2009checklist.doc 